\title{
Electrochemical detection of uric acid via uricase-immobilized graphene oxide
}

\begin{abstract}
Measurement of the uric acid level in the body can be improved by biosensing with respect to the accuracy, sensitivity and time consumption. This study has reported the immobilization of uricase onto graphene oxide $(\mathrm{GO})$ and its function for electrochemical detection of uric acid. Through chemical modification of GO using 1-ethyl-3-(dimethylaminopropyl) carbodiimide (EDC) and N-hydroxysulfosuccinimide (NHS) as cross-linking reagents, the enzyme activity of the immobilized uricase was much comparable to the free enzyme with $88 \%$ of the activity retained. The modified GO-uricase (GOU) was then subjected to electrocatalytic detection of uric acid (UA) via cyclic voltammetry (CV). For that reason, a glassy carbon electrode (GCE) was modified by adhering the GO along with the immobilized uricase to facilitate the redox reaction between the enzyme and the substrate. The modified GOU/GCE outperformed a bare electrode through the electrocatalytic activity with an amplified electrical signal for the detection of UA. The electrocatalytic response showed a linear dependence on the UA concentration ranging from 0.02 to $0.49 \mathrm{mM}$ with a detection limit of $3.45 \mu \mathrm{M}$ at $3 \sigma / \mathrm{m}$. The resulting biosensor also exhibited a high selectivity towards UA in the presence of other interference as well as good reproducibility.
\end{abstract}

Keyword: Uricase; Immobilization; GO; Electrocatalytic detection; Uric acid; Redox reaction 